\title{
REGULADORES VEGETAIS APLICADOS NO SULCO DE PLANTIO EM CULTIVARES DE CANA -DE-AÇÚCAR
}

\section{PLANT REGULATORS APPLIED IN THE PLANTING FURROW IN SOME SUGARCANE CULTIVARS}

\author{
Marina Munhoz Rosato FERREIRA \\ Luiz Henrique Zuculo FERREIRA ${ }^{2}$ \\ Antonio César BOLONHEZI ${ }^{3}$
}

\begin{abstract}
RESUMO
Novas tecnologias de produção tornam-se uma estratégia importante para o incremento da produtividade da canade-açúcar. O presente trabalho teve como objetivo estudar os efeitos da aplicação, no sulco de plantio, de reguladores vegetais (RVs - ácido indol-butírico (análago de auxina) à $0,05 \mathrm{~g} \mathrm{dm}^{-3}$, cinetina (análogo de citocinina) à $0,09 \mathrm{~g} \mathrm{dm}^{-3} \mathrm{e}$ ácido giberélico (análago de giberelina) à $0,05 \mathrm{~g} \mathrm{dm}^{-3}$ ) em oito cultivares de cana-de-açúcar para obter informações daquelas que melhor se adaptam as condições edafoclimáticas da região de Pereira Barreto (SP). O experimento foi instalado na Fazenda Santa Terezinha, localizada no município de Pereira Barreto - SP, em delineamento experimental de blocos casualizados em esquema de parcelas subdivididas, contendo 8 cultivares e quatro repetições (8x2x4). As cultivares foram: SP89-1115, SP813250, SP83-2847, SP91-3011, RB72454, RB867515, RB835054 e SP91-3440 tratadas e não tratadas com RVs. Os resultados permitem concluir que o uso de reguladores vegetais no sulco de plantio nas cultivares SP89-1115, SP83-2847 e SP81-3250 promove o aumento no número de perfilhos, acréscimos no diâmetro de colmo e, portanto, um incremento na produtividade de colmos.
\end{abstract}

Palavras-chave: Saccharum spp, perfilhamento, diâmetro de colmo e produtividade de colmos.

\begin{abstract}
New production Technologies becomes an important strategy to increase the productivity of sugarcane. The present work aimed to study the effects of application, in the planting furrow, of plant regulators (RVs - indolbutiric acid (auxin - related) a $0,05 \mathrm{~g} \mathrm{dm}^{-3}$; kinetin (cytokinin - related) a $0,09 \mathrm{~g} \mathrm{dm}^{-3}$ and giberelic acid (giberelin - related) a $0,05 \mathrm{~g} \mathrm{dm}^{-3}$ ) in eight cultivars of sugarcane to obtain information those that better adapt in the edaphic and climatic conditions of region of Pereira Barreto (state SP). The experiment was conducted at Santa Terezinha Farm, located in Pereira Barreto, state São Paulo, Brazil, in an experimental randomized block design scheme in a split plot containing 8 cultivars and four replications (8x2x4). The cultivars were: SP89-1115, SP81-3250, SP83-2847, SP91-3011, RB72454, RB867515, RB835054 e SP91-3440 treated or not treated with RVs. The results showed that the use of plant regulators in the furrow applied in cultivars SP89-1115, SP83-2847 e SP81 -3250 promotes increase in number of tillers, increase in diameter of stalk and thus an increase on productivity of stalks.

Key-words: Saccharum spp, tillering, diameter of stalk and productivity of stalks.
\end{abstract}

\footnotetext{
${ }^{1}$ Engenheira Agrônoma, Doutoranda em Sistemas de Produção FEIS/UNESP - Ilha Solteira/SP. E-mail: marinamrosato@ig.com.br. 2Engenheiro Agrônomo, Usina Santa Adélia, Pereira Barreto/SP. E-mail: Lzferreira@usinasantaadelia.com.br

${ }^{3}$ Docente do Departamento de Fitotecnia, Tecnologia de Alimentos e Sócio - Economia - FEIS/UNESP - Ilha Solteira/SP. Av. Brasil $n^{\circ}$ 56, centro - C.P. 31 - Ilha Solteira/SP, 15385-000. E-mail: bolonha@agr.feis.unesp.br
} 
FERREIRA, M. M. R. et al. Reguladores vegetais aplicados...

\section{INTRODUÇÃO}

Com a necessidade de redução dos custos de produção, a busca por novas tecnologias de fácil utilização e que possam incrementar a produtividade de colmos e a qualidade tecnológica, tornou-se de vital importância a análise desses processos. Neste sentido, a aplicação de reguladores vegetais sobre os toletes, no momento da cobertura das mudas pode ser uma boa alternativa para estimular uma rápida brotação e contribuir para um maior perfilhamento da cana-de-açúcar. Costa (2007) mencionou que a ação de hormônios vegetais depende de diversos fatores ambientais e do material genético. Silva et al. (2008) reportaram que a resposta da cana-de-açúcar ao regulador vegetal aplicado via foliar em soqueiras depende da variedade e promove aumento na produtividade de colmos. Rosseto et al. (2007) e Vasconcelos et al. (2007) apud Costa (2007), reportaram que o regulador vegetal com auxina, citocinina e giberelina, na canade-açúcar, promoveu aumentos na brotação e na massa seca das raízes, respectivamente. Estes mesmos autores constataram que os efeitos variam de uma variedade para outra. Costa (2007) mencionou que o regulador vegetal com auxina, citocinina e giberelina, o mesmo utilizado neste experimento, promoveram aumentos na produtividade de colmos em diversas cultivares de cana-de-açúcar em vários ambientes. Miguel et al. (2009) concluíram que a aplicação de reguladores vegetais, na dose $0,5 \mathrm{I}$ ha'
1 com aplicação via tolete de cana-de-açúcar na cultivar RB855536 apresentou maior produtividade, maior lucratividade, sendo a forma de aplicação mais economicamente viável.

Por outro lado, Bolonhezi et al. (2006), utilizando o mesmo produto (RVs), reportaram que a cultivar RB835486 cultivada em ambiente caracterizado como estressante mostrou ser não responsiva ao regulador vegetal. Ferreira (2008) concluiu que esta mesma cultivar, em ciclo de segunda soca, também não respondeu ao regulador vegetal aplicado via foliar.

Objetivou-se neste trabalho avaliar oito cultivares de cana-de-açúcar tratadas com um produto a base de $0,09 \mathrm{~g} \mathrm{dm}^{-3}$ de citocinina , $0,05 \mathrm{~g} \mathrm{dm}$ ${ }^{-3}$ de auxina e $0,05 \mathrm{~g} \mathrm{dm}^{-3}$ de giberelina aplicado sobre os toletes durante o plantio.

\section{MATERIAL E MÉTODOS}

O experimento foi instalado numa área de cana-de-açúcar localizada na Fazenda Santa Teresinha no município de Pereira Barreto - SP, com as coordenadas geográficas de, $51^{\circ} 01^{\prime} 39,49^{\prime \prime}$ de longitude oeste e a $20^{\circ} 35^{\prime} 48,74$ " de latitude sul, com altitude de 393 metros. O solo da área é classificado como Argissolo Vermelho eutrófico.

$\mathrm{Na}$ figura 1 apresenta-se a precipitação pluvial mensal e a distribuição de chuvas na área do experimento durante o ciclo da cana planta. A análise química do solo está apresentada na tabela 1.

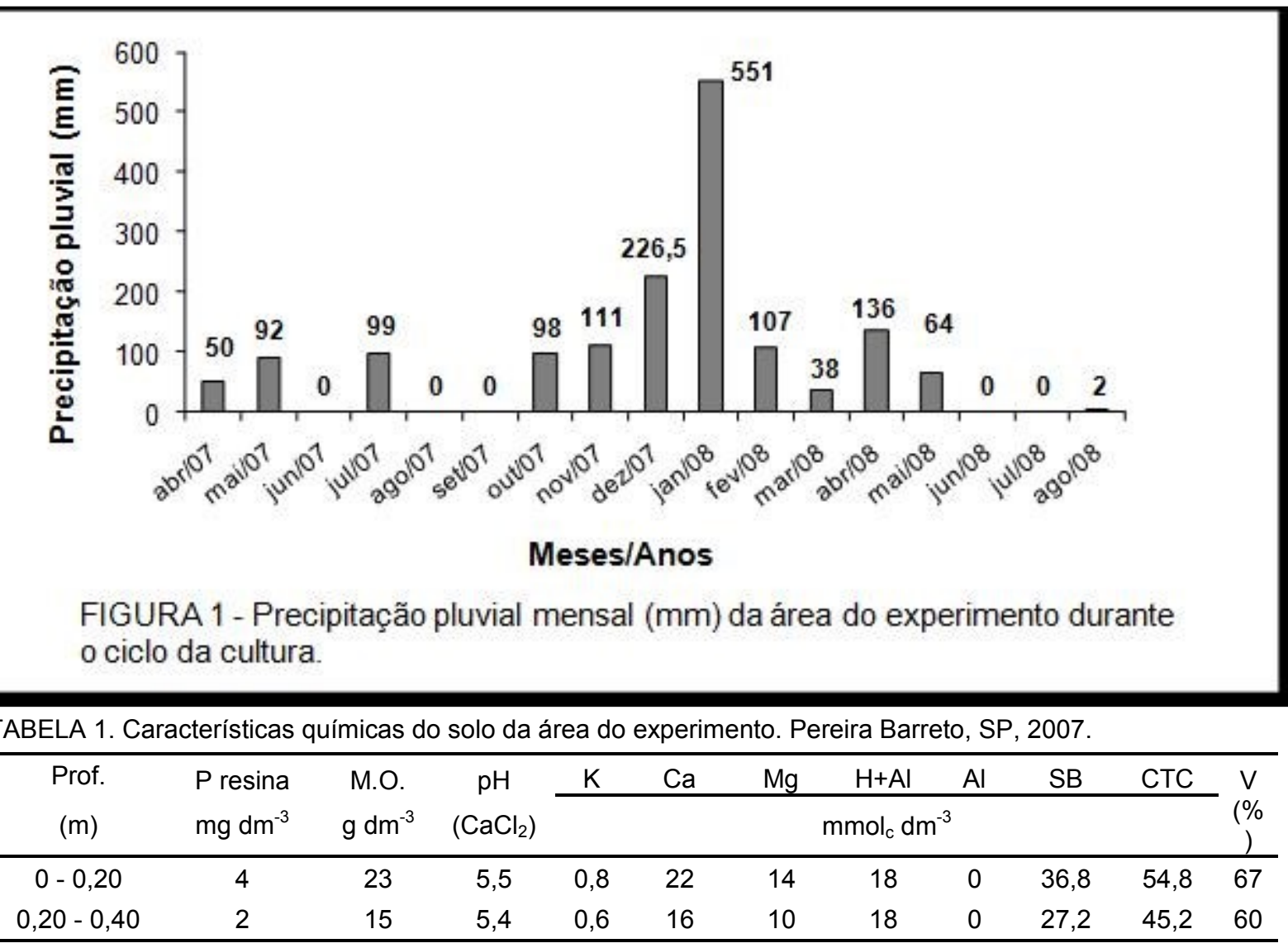

M.O.= matéria orgânica; S.B.= soma de bases; $\mathrm{CTC}=$ capacidade de troca catiônica; $\mathrm{V}=$ saturação de base. 
FERREIRA, M. M. R. et al. Reguladores vegetais aplicados...

O delineamento experimental utilizado foi o de blocos casualizados em esquema de parcelas subdivididas, contendo 8 cultivares e quatro repetições $(8 \times 2 \times 4)$. Cada bloco foi composto por duas parcelas, tratadas e não tradadas com reguladores vegetais (RVs), cada parcela foi dividida em 8 subparcelas, onde foram distribuídas as seguintes cultivares: SP89-1115, SP81-3250, SP83-2847, SP913011, RB72454, RB867515, RB835054 e SP913440. As subparcelas foram constituídas de 5 linhas de $10 \mathrm{~m}$ de comprimento espaçadas de $1,50 \mathrm{~m}$.

As características do produto - reguladores vegetais (RVs) utilizado no experimento são: ácido indol-butírico (análago de auxina) à $0,05 \mathrm{~g} \mathrm{dm}$ ; ; cinetina (análogo de citocinina) à $0,09 \mathrm{~g} \mathrm{dm}^{-3} \mathrm{e}$ ácido giberélico (análago de giberelina) à $0,05 \mathrm{~g} \mathrm{dm}^{-}$

A sulcação foi realizada a $0,40 \mathrm{~m}$ de profundidade. A adubação utilizada no sulco de plantio foi de $620 \mathrm{~kg} \mathrm{ha}^{-1}$ (04-30-10).

O plantio foi feito no dia 23 de março de 2007 com mudas de 11 meses de idade, os colmos inteiros foram distribuídos no sulco seguindo a sequência de base com ápice. Em seguida, fez-se o corte dos colmos a cada 3 gemas, permanecendo 12 gemas viáveis por metro. A aplicação dos RVs foi feita durante a operação de cobertura dos toletes na dose de $0,5 \mathrm{dm}^{3} \mathrm{ha}^{-1}$, sendo realizada por implemento apropriado (cobridor), dotado de bicos para pulverizar sobre os toletes, uma calda de inseticida (fipronil: na dosagem de $0,5 \mathrm{~kg} \mathrm{ha}^{-1}$ do produto comercial).

Os herbicidas utilizados foram o clomazone na dose de $1,8 \mathrm{~kg} \mathrm{ha}^{-1}$ e diuron + hexazinone na dose de $1,5 \mathrm{~kg} \mathrm{ha}^{-1}$.

A colheita do experimento foi realizada no dia 05 de agosto de 2008, o que representa 16 meses de idade, sendo realizada pelo método manual e sem a queima para despalha.

Avaliou-se o perfilhamento (número de perfilhos por metro) aos 22, 29, 35, 70, 100, 141 e 211 dias após plantio (DAP), nas 3 linhas centrais de cada parcela. $O$ diâmetro médio de colmos foi obtido pela medição do diâmetro da base, meio e ponta, em 10 colmos por parcela. O comprimento de colmos foi determinado pela medição da distância entre o colo e a lígula da última folha aberta (folha +1 ). A produtividade de colmos, conforme a metodologia de Landell \& Silva (2004), estima a produtividade de colmos por hectare conforme a equação:

$$
\mathrm{TCH}=\frac{\mathrm{d}^{2} \times \mathrm{C} \times \mathrm{h}(0,007854)}{\mathrm{E}}
$$

Onde: $\mathrm{h}=$ comprimento de colmo $(\mathrm{cm}), \mathrm{C}=$ número de perfilhos por metro, $\mathrm{d}=$ diâmetro de colmos $(\mathrm{cm})$ e espaçamento entre os sulcos $(E)$.

Para as análises tecnológicas (brix, fibra, PC, Pol, AR, pureza e cálculo do ATR), foram colhidos 10 colmos por parcela sem broca. Sendo realizada no laboratório da Usina Santa Adélia - Pereira Barreto/SP, conforme métodos definidos pelo CONSECANA (2003).

Após as avaliações os dados foram tabulados e submetidos à análise de variância; as médias foram comparadas pelo teste Tukey a $5 \%$ de significância, através do programa estatístico SISVAR (Ferreira, 2000).

\section{RESULTADOS E DISCUSSÃO}

Considerando os primeiros 35 DAP como sendo a fase de brotação, observa-se que os RVs favoreceram de modo significativo o estabelecimento inicial da cana (Tabela 2). Nota-se ainda que houve um efeito positivo dos RVs sobre o número de perfilhos por metro até 141 DAP, sendo que, aos 70DAP verifica-se a maior diferença entre os tratamentos com e sem os RVs. Acredita-se que quando as condições de umidade do solo são favoráveis (Figura 1), com os estímulos dos RVs há um maior perfilhamento, por outro lado, quando as plantas estão estressadas pela falta de água, tais estímulos não são assimilados pelas plantas. Cabe ressaltar que não houve efeito significativo da interação RVs e cultivares.

TABELA 2. Médias do número de perfilhos por metro de cultivares de cana-de-açúcar cultivadas com e sem aplicação de reguladores vegetais (RVs) no sulco de plantio. Pereira Barreto, SP, 2007.

\begin{tabular}{|c|c|c|c|c|c|c|c|}
\hline \multirow[t]{2}{*}{$\begin{array}{l}\text { Tratamen- } \\
\text { tos }\end{array}$} & \multicolumn{7}{|c|}{$\begin{array}{c}\text { Perfilhos } \mathrm{m}^{-1} \\
\text { Dias após plantio (DAP) }\end{array}$} \\
\hline & 22 & 29 & 35 & 70 & 100 & 141 & 211 \\
\hline Sem RVs & $6,3 a$ & $7,7 \mathrm{~b}$ & $10,4 b$ & $20,3 b$ & $22,0 a$ & $23,3 b$ & $18,1 \mathrm{a}$ \\
\hline Com RVs & $7,3 a$ & $9,2 a$ & $11,8 a$ & $24,8 a$ & $22,9 a$ & $25,4 a$ & $19,6 a$ \\
\hline DMS & 1,2 & 1,4 & 1,3 & 1,1 & 2,9 & 1,8 & 1,6 \\
\hline CV(\%) & 21,5 & 20,9 & 14,7 & 5,9 & 16,3 & 9,3 & 10,6 \\
\hline
\end{tabular}

Médias seguidas de mesma letra na coluna não diferem entre si pelo teste de Tukey $(p<0,05)$. 
FERREIRA, M. M. R. et al. Reguladores vegetais aplicados...

$\mathrm{Na}$ fase final do perfilhamento, aos 141 DAP, nota-se que os RVs favoreceram um maior número de perfilhos por metro (Tabela 3 ), mas, apenas na cultivar SP99-3440 é que os RVs mos- traram efeito significativo. No entanto, este efeito benéfico no número de perfilhos não se manteve até o final do ciclo, pois o número de colmos não variou entre os tratamentos.

TABELA 3. Médias do número de perfilhos por metro de cultivares de cana-de-açúcar com e sem aplicação de reguladores vegetais (RVs) no sulco de plantio. Pereira Barreto, SP, 2007.

\begin{tabular}{|c|c|c|c|c|c|c|c|c|c|c|c|c|c|c|c|c|}
\hline \multirow{3}{*}{$\begin{array}{l}\text { Cultivares } \\
\text { SP89-1115 }\end{array}$} & \multicolumn{4}{|c|}{$29 \mathrm{DAP}$} & \multicolumn{4}{|c|}{$35 \mathrm{DAP}$} & \multicolumn{4}{|c|}{70 DAP } & \multicolumn{4}{|c|}{$141 \mathrm{DAP}$} \\
\hline & \multicolumn{2}{|c|}{ Com RVs } & \multicolumn{2}{|c|}{ Sem RVs } & \multicolumn{2}{|c|}{ Com RVs } & \multicolumn{2}{|c|}{ Sem RVs } & \multicolumn{2}{|c|}{ Com RVs } & \multicolumn{2}{|c|}{ Sem RVs } & \multicolumn{2}{|c|}{ Com RVs } & \multicolumn{2}{|c|}{ Sem RVs } \\
\hline & 14,36 & $\mathrm{Aa}$ & 11,45 & $\mathrm{Ba}$ & 17,06 & $\mathrm{Aa}$ & 14,65 & $\mathrm{Ba}$ & 22,71 & $A b c$ & 11,66 & $\mathrm{Bc}$ & 19,46 & $\mathrm{Ac}$ & 17,83 & $\mathrm{Ac}$ \\
\hline SP81-3250 & 9,37 & $\mathrm{Bb}$ & 8,06 & $\mathrm{Bb}$ & 14,29 & $\mathrm{Ab}$ & 12,25 & $\mathrm{Bab}$ & 32,50 & $\mathrm{Aa}$ & 25,75 & $\mathrm{Ba}$ & 30,75 & $\mathrm{Ba}$ & 28,00 & $\mathrm{Ba}$ \\
\hline SP83-2847 & 8,24 & $\mathrm{Bb}$ & 6,96 & $\mathrm{Bb}$ & 11,06 & $\mathrm{Bc}$ & 9,90 & $\mathrm{Bbc}$ & 21,12 & $\mathrm{Bc}$ & 18,79 & $\mathrm{Bbc}$ & 23,17 & $\mathrm{Bbc}$ & 23,21 & Babc \\
\hline SP91-3011 & 8,32 & $\mathrm{Bb}$ & 7,67 & $\mathrm{Bb}$ & 9,91 & Bcd & 9,20 & $\mathrm{Bc}$ & 26,54 & $A b c$ & 21,54 & Bab & 27,96 & Bab & 24,79 & $\mathrm{Bab}$ \\
\hline RB72454 & 8,24 & $\mathrm{Bb}$ & 7,28 & $\mathrm{Bb}$ & 10,21 & $\mathrm{Bc}$ & 9,37 & $\mathrm{Bc}$ & 23,04 & $\mathrm{Bbc}$ & 21,45 & Bab & 23,33 & $\mathrm{Bbc}$ & 24,87 & Bab \\
\hline RB867 & 10,31 & $\mathrm{Ab}$ & 7,81 & $\mathrm{Bb}$ & 14,00 & $\mathrm{Ab}$ & 12,15 & Bab & 24,17 & $\mathrm{Bbc}$ & 20,71 & Babc & 23,25 & $\mathrm{Bbc}$ & 21,00 & $\mathrm{Bbc}$ \\
\hline RB835 & 9,87 & $\mathrm{Ab}$ & 8,06 & $\mathrm{Bb}$ & 10,59 & $\mathrm{Bc}$ & 9,80 & $\mathrm{Bbc}$ & 26,83 & $A b$ & 21,66 & $\mathrm{Ba}$ & 27,04 & Bab & 23,79 & Bab \\
\hline SP91-3440 & 5,19 & $\mathrm{Bc}$ & 4,48 & $\mathrm{Bc}$ & 7,48 & $\mathrm{Bd}$ & 6,26 & $\mathrm{Bd}$ & 21,21 & $\mathrm{Ac}$ & 16,87 & $\mathrm{Bbc}$ & 28,04 & $A a b$ & 22,58 & $\mathrm{Bbc}$ \\
\hline DMS $(\mathrm{V})^{*}$ & \multicolumn{2}{|c|}{2,40} & \multicolumn{2}{|c|}{2,40} & \multicolumn{2}{|c|}{2,67} & \multicolumn{2}{|c|}{2,67} & \multicolumn{2}{|c|}{5,51} & \multicolumn{2}{|c|}{5,51} & \multicolumn{2}{|c|}{5,39} & \multicolumn{2}{|c|}{5,39} \\
\hline $\operatorname{DMS}(T)^{* *}$ & \multicolumn{2}{|c|}{1,47} & \multicolumn{2}{|c|}{1,47} & \multicolumn{2}{|c|}{1,68} & \multirow{2}{*}{\multicolumn{2}{|c|}{$\begin{array}{c}1,68 \\
1472\end{array}$}} & \multicolumn{2}{|c|}{3,49} & \multicolumn{2}{|c|}{3,49} & \multicolumn{2}{|c|}{3,41} & \multicolumn{2}{|c|}{3,41} \\
\hline CV (\%) & \multicolumn{2}{|c|}{20,87} & \multicolumn{2}{|c|}{20,87} & \multicolumn{2}{|c|}{14,72} & & & \multicolumn{2}{|c|}{5,93} & 5, & & 9 & & & \\
\hline
\end{tabular}

*DMS (Variedades); ** DMS (Tratamentos)

Médias seguidas de mesma letra não diferem entre si pelo teste de Tukey $(p<0,05)$. Linha letra maiúscula e coluna letra minúscula.

Os reguladores vegetais não afetaram o comprimento do colmo nem a massa de um colmo.

$\mathrm{O}$ aumento de produtividade se deve aos

RVs possibilitarem aumento do diâmetro de colmo e não sobre as demais variáveis analisadas (Tabela 4).

TABELA 4. Médias das variáveis diâmetro de colmo ( $\mathrm{cm})$, comprimento de colmo $(\mathrm{m})$, massa de um colmo $(\mathrm{kg})$, número de colmos por metro e produtividade de colmos (tonelada de cana por hectare) de cultivares de canade-açúcar cultivadas com e sem aplicação de reguladores vegetais (RVs) no sulco de plantio. Pereira Barreto, SP, 2008.

\begin{tabular}{cccccc}
\hline Tratamentos & $\begin{array}{c}\text { Diâmetro de } \\
\text { colmo }(\mathrm{cm})\end{array}$ & $\begin{array}{c}\text { Comprimento } \\
\text { de colmo }(\mathrm{m})\end{array}$ & $\begin{array}{c}\text { Massa de } 1 \\
\text { colmo }(\mathrm{kg})\end{array}$ & $\begin{array}{c}\mathrm{N}^{\circ} \text { de } \\
\text { colmos m }^{-1}\end{array}$ & $\begin{array}{c}\text { Produtividade de } \\
\text { colmos }\left(\mathrm{t} \mathrm{ha}^{-1}\right)\end{array}$ \\
\hline Sem RVs & $2,75 \mathrm{~b}$ & $2,488 \mathrm{a}$ & $1,53 \mathrm{a}$ & $9,61 \mathrm{a}$ & $95,45 \mathrm{~b}$ \\
Com RVs & $2,89 \mathrm{a}$ & $2,483 \mathrm{a}$ & $1,62 \mathrm{a}$ & $9,44 \mathrm{a}$ & $102,74 \mathrm{a}$ \\
\hline DMS & 0,049 & 0,152 & 0,201 & 0,843 & 6,33 \\
CV(\%) & 2,22 & 7,69 & 16,07 & 11,12 & 8,03 \\
\hline
\end{tabular}

Médias seguidas de mesma letra não diferem entre si pelo teste de Tukey $(p<0,05)$.

Cabe destacar que algumas cultivares mostraram uma maior sensibilidade aos RVs (Tabela 5), como é o caso da SP83-2847 e da SP81-3250, que tiveram as maiores médias de produtividades de colmos quando tratadas com os RVs, muito embora, as médias não tenham apresentado diferença signi- ficativa. Este resultado confirma relatos de Costa (2007) e Silva et al. (2008), mas contradizem Bolonhezi et al. (2006) e Ferreira (2008) que mencionaram não existir efeito benéfico do regulador vegetal sobre a produtividade de colmos em cana cultivada em ambientes estressantes. 
FERREIRA, M. M. R. et al. Reguladores vegetais aplicados...

TABELA 5. Diâmetro do colmo $(\mathrm{cm})$ e produtividade $\left(\mathrm{t} \mathrm{ha}{ }^{-1}\right)$ de cultivares de cana-de-açúcar com e sem aplicação de reguladores vegetais (RVs) no sulco de plantio. Pereira Barreto, SP, 2008.

\begin{tabular}{|c|c|c|c|c|}
\hline \multirow{3}{*}{$\begin{array}{c}\text { Cultivares } \\
\text { SP91-3440 }\end{array}$} & \multicolumn{2}{|c|}{ Diâmetro de colmo (cm) } & \multicolumn{2}{|c|}{ Produtividade de colmos $\left(\mathrm{t} \mathrm{ha}^{-1}\right)$} \\
\hline & Com RVs & Sem RVs & Com RVs & Sem RVs \\
\hline & 2,85 Aab & $2,70 \mathrm{Aa}$ & 101,5 Aabc & 99,06 Aab \\
\hline SP89-1115 & $3,07 \mathrm{Aa}$ & $2,77 \mathrm{Ba}$ & 87,71 Acd & $77,07 \quad \mathrm{Ab}$ \\
\hline RB867515 & 3,02 Aab & $2,92 \mathrm{Aa}$ & 124,68 Aa & 123,15 Aa \\
\hline SP83-2847 & $2,82 \mathrm{Aab}$ & $2,60 \mathrm{Aa}$ & 104,87 Aabc & $84,59 \mathrm{Ab}$ \\
\hline RB72454 & 3,00 Aab & $2,90 \mathrm{Aa}$ & $110,62 \mathrm{Aab}$ & 104,93 Aab \\
\hline SP91-3011 & 2,72 Aab & $2,65 \mathrm{Aa}$ & 96,16 Aabc & 97,57 Aab \\
\hline RB835054 & $2,65 \mathrm{Ab}$ & $2,72 \mathrm{Aa}$ & 73,58 Ac & $75,36 \mathrm{Ab}$ \\
\hline SP81-3250 & 2,95 Aab & $2,75 \mathrm{Aa}$ & $122,83 \mathrm{Aa}$ & 101,89 Aab \\
\hline $\mathrm{DMS}(\mathrm{V}){ }^{*}$ & 0,377 & 0,377 & 34,948 & 34,948 \\
\hline $\operatorname{DMS}(T)^{* *}$ & 0,239 & 0,239 & 22,137 & 22,137 \\
\hline $\mathrm{CV}(\%)$ & 2,22 & 2,22 & 8,03 & 8,03 \\
\hline
\end{tabular}

* DMS (Variedades); **DMS (Tratamentos)

Médias seguidas de mesma letra não diferem entre si pelo teste de Tukey $(p<0,05)$. Linha letra maiúscula e coluna letra minúscula.

É possível que os efeitos positivos deste regulador vegetal sobre o desenvolvimento da canade-açúcar e consequente incremento na produtividade de colmos, sejam mais comuns em ambientes de produção "A ou B", cujos solos são de maior fertilidade, principalmente com relação aos teores de cálcio e fósforo. Especula-se, deste modo, que as plantas tratadas via tolete, depois de assimilarem o estímulo inicial do regulador vegetal, encontram uma condição química do solo favorável ao bom desenvolvimento radicular, enquanto que, nos ambientes considerados estressantes, com teores de cálcio e fósforo baixos e a presença de alumínio tóxico, tem uma condição química do solo que dificulta o crescimento radicular. Assim, a planta tendo volume radicular limitado, absorve menos água e nutrientes, além de haver uma diminuição na síntese de citocininas, hormônios importantes para o crescimento da planta. Ou seja, é possível que os efeitos positivos dos RVs sobre o desenvolvimento radicular sejam insuficientes para um maior crescimento radicular nos solos menos férteis com baixos teores de cálcio e fósforo. Como pode ser observado na Tabela 1, o solo da área experimental possui altos teores de cálcio e magnésio, porém, baixo teor de fósforo e ambiente de produção " $C$ ".

Das características que compõem a qualidade tecnológica da cana, verifica-se que os RVs reduziram a porcentagem dos açúcares redutores, mas não influenciaram as demais características (Tabela 6) concordando com Bolonhezi et al. (2006), Ferreira (2008) e Silva (2008). Como os RVs estimularam um aumento no diâmetro dos colmos, as plantas utilizaram os açúcares (glicose e frutose) para o crescimento, reduzindo, portanto, os teores destes açúcares no parênquima de acúmulo.

TABELA 6. Médias das variáveis tecnológicas (Brix\%caldo, Fibra\%cana, PC\%cana, Pol\%caldo, AR\%cana, Pureza (\%)) e ATR (kg de açúcar $\mathrm{t}^{-1}$ de cana)) de cultivares de cana-de-açúcar cultivadas com e sem aplicação de reguladores vegetais (RVs) no sulco de plantio. Pereira Barreto, SP, 2008.

\begin{tabular}{|c|c|c|c|c|c|c|c|}
\hline \multirow{2}{*}{ Tratamentos } & Brix & Fibra & PC & Pol & AR & Pureza & \multirow{2}{*}{$\begin{array}{c}\text { ATR } \\
\mathrm{kg} \mathrm{t}^{-1} \text { cana }\end{array}$} \\
\hline & \multicolumn{6}{|c|}{$\%$} & \\
\hline Sem RVs & $23,06 a$ & $14,70 a$ & $16,69 a$ & $20,76 a$ & $0,52 a$ & $90,02 a$ & $162,81 a$ \\
\hline Com RVs & $22,39 a$ & $14,46 a$ & $16,37 a$ & $20,26 a$ & $0,40 b$ & $90,47 a$ & $158,83 a$ \\
\hline DMS & 1,59 & 0,55 & 0,905 & 1,63 & 0,10 & 0,56 & 8,86 \\
\hline CV (\%) & 8,79 & 4,76 & 6,88 & 10,02 & 27,92 & 0,78 & 6,93 \\
\hline
\end{tabular}

Médias seguidas de mesma letra não diferem entre si pelo teste de Tukey $(p<0,05)$. 
FERREIRA, M. M. R. et al. Reguladores vegetais aplicados...

\section{CONCLUSÕES}

Os reguladores vegetais estimulam o perfilhamento em algumas cultivares.

Os reguladores vegetais aumentam o diâmetro do colmo e, em consequência, incrementam a produtividade de colmos. aos RVs.

A cultivar SP81-3250 é a mais responsiva

Os RVs são uma tecnologia que pode ser utilizada para o manejo específico de uma cultivar.

\section{REFERÊNCIAS}

1. BOLONHEZI, A. C. et al. Doses de fósforo associadas com regulador vegetal em cana-de-açúcar (Saccharum spp), variedade RB835486. Reunião Brasileira de fertilidade do solo e nutrição de plantas, 27. Bonito, 2006. CD-ROM.

2. CONSELHO DOS PRODUTORES DE CANA-DE-AÇÚCAR, AÇÚCAR E ÁLCOOL DO ESTADO DE SÃO PAULO (CONSECANA). Manual de Instruções. 4 ed. Piracicaba: Opinião, 2003. 116p.

3. COSTA, A. G. F. Manejo Fisiológico da cana-de-açúcar. Stoller do Brasil. Semana da agronomia. Ilha Solteira, 2007. CD-ROM.

4. FERREIRA, D.F. Análises estatísticas por meio do SISVAR para Windows versão 4.0. In: REUNIÃO ANUAL DA REGIÃO BRASILEIRA DA SOCIEDADE INTERNACIONAL DE BIOMETRIA, 45., 2000, São Carlos-SP. Programas e Resumos... São Carlos: UFSCar, 2000. p.235

5. FERREIRA, G. S. Reguladores vegetais associados à micronutrientes aplicados via foliar em cana soca da variedade RB835486. 2008. 21f. Trabalho de Graduação - Faculdade de Engenharia de Ilha Solteira - Unesp, Ilha Solteira, 2008.

6. LANDELL, M.G.A.; SILVA, M.A. As estratégias de seleção da cana em desenvolvimento no Brasil. Visão Agrícola, v. 1, n. 1, p. 18-23, 2004

7. MIGUEL, F. B. et al. Viabilidade econômica na utilização de um regulador vegetal em cana-planta. Informações Econômicas, v. 39, n. 1, p. 53-59, 2009.

8. ROSSETTO, R. et al. Efeito de biorregulador e de fertilizantes aplicados no plantio da cana-de-açúcar. In: CONGRESSO BRASILEIRO DE FISIOLOGIA VEGETAL, 11., 2007, Gramado, RS. Resumo... Gramado: Sociedade Brasileira de Fisiologia Vegetal, 2007.

9. SILVA, A. R. B. et al. Avaliação da aplicação de Stimulate ${ }^{\circledR}$ no sulco de plantio de variedades de cana-de-açúcar. In: SIMPÓSIO BRASILEIRO SOBRE ECOFISIOLOGIA, MATURAÇÃO E MATURADORES EM CANA DE AÇÚCAR, 1., 2008, Botucatu, SP. Anais... Botucatu: UNESP, 2008. p. 116-120.

10.TAIZ, L.; ZEIGER, E. Fisiologia Vegetal. 3.ed. Porto Alegre: Artmed, 2006, 719p. 